\title{
Limitations of effective medium theory in multilayer graphite/hBN heterostructures
}

\author{
Petersen, René; Pedersen, Thomas Garm; Gjerding, Morten Niklas; Thygesen, Kristian Sommer
}

Published in:

Physical Review B

Link to article, DOI:

10.1103/PhysRevB.94.035128

Publication date:

2016

Document Version

Publisher's PDF, also known as Version of record

Link back to DTU Orbit

Citation (APA):

Petersen, R., Pedersen, T. G., Gjerding, M. N., \& Thygesen, K. S. (2016). Limitations of effective medium theory in multilayer graphite/hBN heterostructures. Physical Review B, 94(3), [035128].

https://doi.org/10.1103/PhysRevB.94.035128

\section{General rights}

Copyright and moral rights for the publications made accessible in the public portal are retained by the authors and/or other copyright owners and it is a condition of accessing publications that users recognise and abide by the legal requirements associated with these rights.

- Users may download and print one copy of any publication from the public portal for the purpose of private study or research.

- You may not further distribute the material or use it for any profit-making activity or commercial gain

- You may freely distribute the URL identifying the publication in the public portal 


\title{
Limitations of effective medium theory in multilayer graphite/hBN heterostructures
}

\author{
René Petersen* and Thomas Garm Pedersen \\ Department of Physics and Nanotechnology, Aalborg University, DK-9220 Aalborg East, Denmark \\ and Center for Nanostructured Graphene (CNG), DK-9220 Aalborg East, Denmark \\ Morten Niklas Gjerding and Kristian Sommer Thygesen \\ Institute of Physics, Technical University of Denmark (DTU), DK-2800 Kgs. Lyngby, Denmark \\ and Center for Nanostructured Graphene (CNG), DK-2800 Kgs. Lyngby, Denmark \\ (Received 3 March 2016; revised manuscript received 2 June 2016; published 12 July 2016)
}

\begin{abstract}
We apply effective medium theory (EMT) to metamaterials consisting of a varying number of consecutive sheets of graphene and hexagonal boron nitride, and compare this with a full calculation of the permittivity and the reflection based on the tight binding method and the transfer matrix method in order to study the convergence to EMT. We find that convergence is reached for both in-plane and out-of-plane directions already for five sheets but that for $\approx 30$ sheets multiple reflection effects causes the reflection spectrum to differ from EMT. We show that modes that are evanescent in air are extremely sensitive to the electronic details of the sheets near the structure boundary and that EMT estimates poorly the reflection of these modes, causing an overestimation of the Purcell factor. Finally, we offer an improved EMT, which gives far better convergence in the low-energy regime.
\end{abstract}

DOI: 10.1103/PhysRevB.94.035128

\section{INTRODUCTION}

Hyperbolic metamaterials (HMMs) have recently attracted much attention due to their interesting applications including hyperlenses and lifetime engineering [1-5]. Of particular interest are graphene based HMMs because of the possibility of exploiting the semimetallic nature of graphene in tuning of the optical properties in the $\mathrm{THz}$ regime via gating or doping [6,7]. Due to the subwavelength nature of these HMMs it has become common practice to homogenize the structure using effective medium theory (EMT) in order to facilitate a simpler description and focus on the HMM properties [5,810]. Experimental studies have confirmed the validity of EMT in $\mathrm{Au} / \mathrm{MgF}_{2}, \mathrm{Ag} / \mathrm{MgF}_{2}$, and $\mathrm{Au} / \mathrm{Al}_{2} \mathrm{O}_{3}$ heterostructures [5,11], but EMT is known to describe incorrectly the reflection of evanescent modes [12] and to fail describing even deep subwavelength structures in some cases [13]. EMT ignores effects near the boundaries between layers that may affect the electronic structure of the constituents and thereby the local refractive index, and the phase shifts introduced as light propagates through individual layers are treated in an average sense. For these reasons, it is important to investigate the accuracy and understand the limitations of EMT, also in the limit of extremely thin layers. In this work, we apply EMT to periodic and finite layered graphite-hexagonal boron nitride $(\mathrm{Gr}-\mathrm{hBN})$ heterostructures with a varying number of consecutive graphene/hBN sheets (see Fig. 1) and calculate the permittivity and the reflection. These structures constitute a strongly anisotropic system sufficiently simple to allow for modeling of the individual constituents as well as the full combined system using tight binding (TB), making Gr-hBN ideal to assess the validity of EMT in both in-plane and out-of-plane directions. We base our EMT calculations on the readily accessible permittivities of graphite and bulk $\mathrm{hBN}$ as well as graphene and monolayer hBN. We investigate periodic

\footnotetext{
*rp@nano.aau.dk
}

structures of the form $N \times \mathrm{Gr}-M \times \mathrm{hBN}$ where $N$ and $M$ denote the number of graphene and hBN sheets in the unit cell, but restrict our studies to structures for which $M=N$ for simplicity. In the following we will denote such periodic structures by $\{N, N\}$, not to be confused with the notation $(N, N)$, which will be introduced later for finite structures. We have checked that our conclusions hold also for asymmetric structures for which $M \neq N$, but in the few cases where major differences arise, we provide an explicit discussion hereof.

In EMT, a uniaxial stratified periodic metamaterial consisting of a metal and a dielectric can be characterized by the effective diagonal permittivity tensor $\varepsilon^{\mathrm{EMT}}=$ diag $\left(\varepsilon_{\|}^{\mathrm{EMT}}, \varepsilon_{\|}^{\mathrm{EMT}}, \varepsilon_{\perp}^{\mathrm{EMT}}\right)$, with effective components given by [4]

$$
\begin{gathered}
\varepsilon_{\|}^{\mathrm{EMT}}=\rho \varepsilon_{\|, \mathrm{m}}+(1-\rho) \varepsilon_{\|, \mathrm{d}}, \\
\frac{1}{\varepsilon_{\perp}^{\mathrm{EMT}}}=\frac{\rho}{\varepsilon_{\perp, \mathrm{m}}}+\frac{1-\rho}{\varepsilon_{\perp, \mathrm{d}}},
\end{gathered}
$$

where $\quad \boldsymbol{\varepsilon}_{m}=\operatorname{diag}\left(\varepsilon_{\|, \mathrm{m}}, \varepsilon_{\|, \mathrm{m}}, \varepsilon_{\perp, \mathrm{m}}\right) \quad$ and $\quad \boldsymbol{\varepsilon}_{d}=\operatorname{diag}\left(\varepsilon_{\|, \mathrm{d}}\right.$, $\left.\varepsilon_{\|, \mathrm{d}}, \varepsilon_{\perp, \mathrm{d}}\right)$ are the permittivity tensors of the metal and the dielectric and $\rho$ is the fill fraction of metal, which will be $\frac{1}{2}$ in all our calculations. By parallel $(\|)$ we refer to the in-plane component (perpendicular to the optical axis) and by perpendicular $(\perp)$ to the out-of-plane component (parallel to the optical axis). We will refer to a single atomic monolayer of graphene or $\mathrm{hBN}$ within the structure as a sheet and to a stack of identical sheets as a layer. Thus, Fig. 1(b) shows four layers and twelve sheets. When referring to the graphitelike or the bulk-hBN-like parts of the structure we will do so by writing $\mathrm{Gr}$ or hBN, respectively. It would be incorrect to refer to the graphitelike part as graphite or graphene since the presence of the $\mathrm{hBN}$ changes its properties.

To assess how well EMT describes the properties of the structure we calculate the permittivity and the reflection spectrum. For the parallel case we compare directly the permittivity calculated using a full model with the EMT permittivity, while for the perpendicular case we calculate the 
(a)

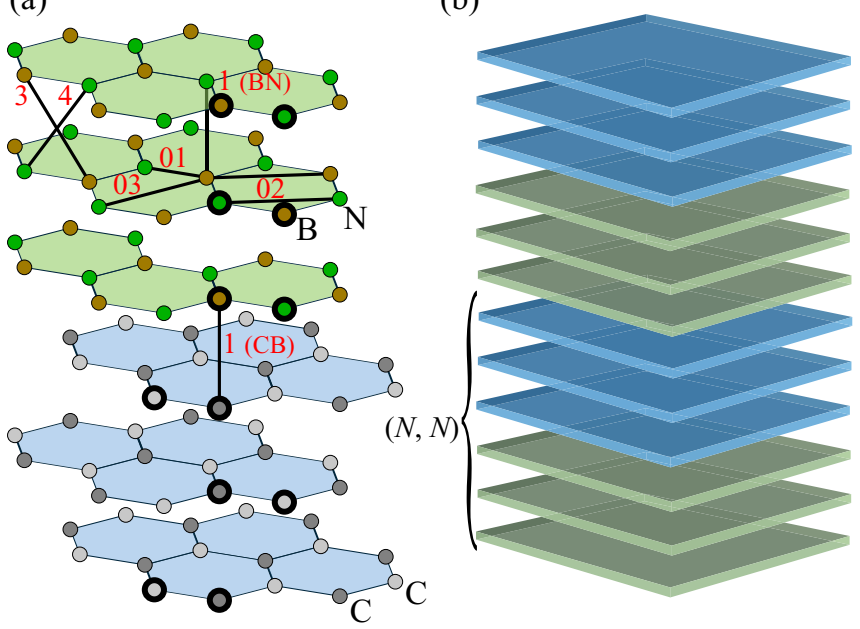

FIG. 1. (a) Model of the periodic structure used for atomistic calculations with coupling constants indicated by red numbers and the atoms of the unit cell marked with thick stroke. The shown structure is denoted by $\{3,3\}(3 \times \mathrm{Gr}-3 \times \mathrm{hBN})$. Notice that graphite is $\mathrm{AB}$ stacked while hBN is $\mathrm{AA}^{\prime}$ stacked. The interlayer distance is $3.35 \AA$. (b) Schematic of the layered structure used for reflection calculations. $(N, N)$ denotes the finite building block of the stack which for the case shown is $(3,3)$. We denote the entire 12-sheet stack shown by $2 \times(3,3)$.

permittivity of each sheet of the unit cell and employ the transfer matrix method (TMM) to calculate the reflection in the full model and in EMT, in order to account for the field variation throughout the structure. We expect the permittivity of $\{N, N\}$ structures to converge to the bulk-based EMT value as the number of sheets increases. On the other hand, as the layers become thicker we no longer satisfy the condition for EMT that the period of the structure is much larger than the wavelength, especially for large $k$ wave vectors. Thus, we expect EMT to work well in a certain range of the number of sheets.

Since many applications of graphene-based HMMs rely on the ability to tune the properties by doping, we investigate in detail how doping of the structure influences the convergence and we provide a simple improvement of EMT to better describe doped structures. Finally, we calculate Purcell factors to see how EMT performs in applications relying on lifetime engineering.

\section{METHODS}

We use a nonorthogonal $\pi$-electron TB model to calculate eigenenergies and eigenmodes of the structure shown in Fig. 1(a), i.e., we solve

$$
\mathbf{H} \cdot \boldsymbol{c}=E \mathbf{S} \cdot \boldsymbol{c}
$$

using standard methods. For the Gr part we use the DFT-LDA fitted TB parameters suggested by Grüneis et al. [16] while for the hBN part we use our own fit to a DFT band structure by employing a least-squares technique. We fit all four $\pi$ bands along the line $\Gamma-M-K-H-L-A[16]$ of the irreducible Brillouin zone (BZ) starting $\frac{6}{7}$ along the line $\Gamma-M$ and ending $\frac{1}{7}$ along the line $L-A$, effectively including only the parts of the band structure that contribute to optical transitions below $\approx 8 \mathrm{eV}$. The zero point of energy in Eq. (3) is chosen as the graphene Dirac point such that for all calculations $E_{F}=0.0 \mathrm{eV}$ corresponds to undoped structures.

DFT band structures and optical response have been calculated using the electronic structure code GPAW [17]. We use a plane-wave cutoff energy of $600 \mathrm{eV}$ and a $\Gamma$-centered Monkhorst-Pack $k$-point sampling of size $30 \times 30 \times 10$ and $120 \times 120 \times 38$ in ground-state calculations for the band structure and for the response calculations, respectively. For graphite, we use a higher $k$-point sampling of $60 \AA$ in the vicinity of the edge from the $K$ to $H$ points of the BZ. We use a Fermi smearing of $25 \mathrm{meV}$. The local density approximation (LDA) was chosen as exchange-correlation functional since it is not expected to influence the conclusions compared to other functionals [18]. The optical response has been calculated in the linear response regime [19] including local field effects using a cutoff energy of $60 \mathrm{eV}$, at which the local field effects were found to be converged, and a level broadening of $5 \mathrm{meV}$. Excitonic effects are ignored. The BZ integrations are done using the linear tetrahedron integration method [20].

In all calculations we use the graphite lattice parameters, that is, $a_{0}=2.46 \AA$ for the lattice constant and $c_{0}=3.35 \AA$ for the sheet distance [16]. Experimental and $a b$ initio studies have found the lattice constant of bulk hBN to be $2 \%$ greater than that of graphite and the sheet distance to be $3.33 \AA$ [21,22], but as an approximation we ignore these differences since they are expected to have no significant influence on our results. We use $\mathrm{AA}^{\prime}$ stacking for the hBN part (boron over nitrogen) [22] and AB stacking (boron over carbon) for the Gr-hBN interface [23], and assume that the $\mathrm{Gr}$ and $\mathrm{hBN}$ layers are not rotated with respect to each other.

The hBN TB parameters are given in Table I, where the notation corresponds to Fig. 1(a). We have chosen our conventions for couplings in $\mathrm{hBN}$ to be similar to those of Ref. [16] for graphite, except for $\gamma_{3}$ and $\gamma_{4}$ where the interpretation is slightly different.

We calculate the permittivity by: (i) calculating the real part of the interband conductivity $\sigma_{\text {inter }}(\omega)$ in the zero-broadening limit; (ii) folding this with a Lorentzian to reintroduce broadening; (iii) doing a Kramers-Kronig transformation to find the imaginary part; (iv) adding the complex intraband conductivity $\tilde{\sigma}_{\text {intra }}(\omega)$; and finally, (v) determining the permittivity from $\varepsilon=\varepsilon_{\infty}+i \tilde{\sigma}(\omega) / \varepsilon_{0} \omega$ [24], where $\epsilon_{\infty}$ is treated as a fitting parameter accounting for $\sigma$ electrons and $\tilde{\sigma}(\omega)$ is the complex conductivity. In all spectra, unless otherwise stated, the

TABLE I. hBN TB parameters. Onsite energies and hopping elements ( $E$ and $\gamma$, respectively) are specified in $\mathrm{eV}$, while overlap integrals $(s)$ are unitless. The carbon-boron coupling $\gamma_{1}^{\mathrm{CB}}$ is taken from [14].

\begin{tabular}{lll}
\hline$E_{0}^{\mathrm{B}}=1.6026$ & $E_{0}^{\mathrm{N}}=-2.9180$ & $\gamma_{01}=-2.5989$ \\
$\gamma_{02}^{\mathrm{BB}}=-0.17068$ & $\gamma_{02}^{\mathrm{NN}}=-0.37578$ & $\gamma_{03}=-0.4056$ \\
$\gamma_{1}^{\mathrm{BN}}=0.13009$ & $\gamma_{3}=0.57442$ & $\gamma_{4}=-0.16411$ \\
$\gamma_{1}^{\mathrm{CB}}=0.4300$ & $s_{01}=0.07760$ & $s_{02}^{\mathrm{BB}}=0.04588$ \\
$s_{02}^{\mathrm{NN}}=0.065273$ & $s_{03}=0.052306$ & $s_{1}=-0.10355$ \\
\hline
\end{tabular}


broadening is set to $50 \mathrm{meV}$ and the thermal energy to $25 \mathrm{meV}$. The real part of the interband conductivity is found using the first-order perturbation theory result [24,25]

$$
\sigma_{\text {inter }}(\omega)=\frac{e^{2}}{4 \pi^{2} \hbar^{2} \omega} \sum_{m} \int_{\mathrm{BZ}} f_{m n} M_{m n} \delta\left(E_{n m}-\hbar \omega\right) d^{3} k,
$$

where $E_{n m}=E_{n}-E_{m}$ is the energy difference between band $n$ and $m, f_{m n}=f\left(E_{m}\right)-f\left(E_{n}\right)$ is the Fermi occupation factor, and $M_{m n}=\left|P_{m n}\right|^{2}$ are the absolute squared momentum matrix elements (MMEs) given by either

$$
M_{\|, m n}=\frac{1}{2}\left|\left\langle\Psi_{m}\left|\frac{\partial \hat{H}}{\partial k_{x}}\right| \Psi_{n}\right\rangle\right|^{2}+\frac{1}{2}\left|\left\langle\Psi_{m}\left|\frac{\partial \hat{H}}{\partial k_{y}}\right| \Psi_{n}\right\rangle\right|^{2}
$$

for the parallel component of the conductivity, or

$$
M_{\perp, m n}=\left|\left\langle\Psi_{m}\left|\frac{\partial \hat{H}}{\partial k_{z}}\right| \Psi_{n}\right\rangle\right|^{2}
$$

for the perpendicular component, with $\Psi$ being the wave function of the system constructed from the eigenvectors found from Eq. (3) and the atomic $\pi$ wave functions. The MME for the parallel component has been symmetrized in $x$ and $y$ to allow the $\boldsymbol{k}$ integration to be done over the irreducible BZ only [25].

The intraband conductivity is modeled as a Drude term $\tilde{\sigma}_{\text {intra }}(\omega)=i \varepsilon_{0} \omega_{p}^{2} /(\omega+i \Gamma)$ where $\Gamma$ is the broadening and $\omega_{p}$ is the plasma frequency given by

$$
\omega_{p}^{2}=\frac{-e^{2}}{4 \pi^{3} \hbar^{2} \varepsilon_{0}} \sum_{n} \iint_{\mathrm{BZ}} M_{n n} \delta\left(E_{n}-E\right) d^{3} k f^{\prime}(E) d E .
$$

In Fig. 2, we present the parallel and perpendicular permittivity of bulk hBN and graphite calculated using both DFT and TB. To match the DFT calculations, the real part of the TB spectrum for bulk hBN has been shifted by an additive

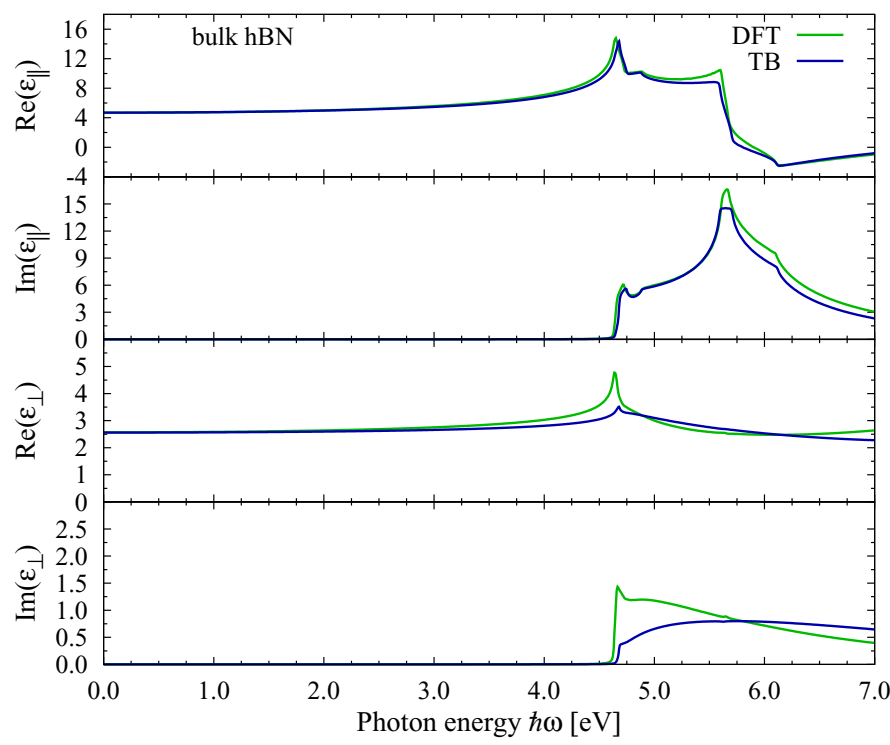

constant of 1.70 for the parallel component and by 1.15 for the perpendicular component, in order to account for energetically distant transitions due to $\sigma$ electrons that are not included in our TB model $[25,26]$. For the parallel component the real and imaginary parts are in excellent agreement with DFT, while the perpendicular component is less well reproduced by TB due to the distribution of $k$ points in the fitting procedure favoring the more important in-plane parameters. For graphite we shift the real part of the parallel permittivity by 1.1 to match the spectrum measured by Taft and Philipp [15]. For the perpendicular component contradicting values of the permittivity are reported in the literature [27] and we therefore shift the TB curve by 1.62 to match our DFT calculations. Excellent agreement between DFT and TB is found, except for low energies due to difficulties with obtaining converged results in DFT in this regime. We observe also good agreement with experiment for the parallel component. The disagreement around $4.25 \mathrm{eV}$ is partly explained by broadening such that increasing the broadening in our spectra gives better agreement (not shown).

Equation (4) is accurate for homogeneous materials such as bulk hBN described above, in which local field effects due to atomic-scale variations can be ignored, and the driving field considered to be approximately constant throughout the entire structure. For inhomogeneous materials such as $\mathrm{Gr} / \mathrm{hBN}$ heterostructures, however, this approximation applies only to the parallel field component $\mathcal{E}_{\|}$, for which the electromagnetic boundary condition states that $\mathcal{E}_{\|, 1}=\mathcal{E}_{\|, 2}$ with 1 and 2 referring to the two sides of a boundary. The perpendicular field component $\mathcal{E}_{\perp}$ varies throughout the heterostructure as can be seen from the boundary condition $\varepsilon_{\perp, 1} \mathcal{E}_{\perp, 1}=\varepsilon_{\perp, 2} \mathcal{E}_{\perp, 2}$ [3]. Given that $\varepsilon_{\perp, 1} \neq \varepsilon_{\perp, 2}$, we will have also $\mathcal{E}_{\perp, 1} \neq \mathcal{E}_{\perp, 2}$.

To include the field variation we calculate the contribution to the permittivity from each sheet of an $\{N, N\}$ structure, from here referred to as the projected permittivity, and use the TMM to calculate the reflection from finite stacks consisting of $S$ sheets in an ambient of air in the configurations $\frac{S}{2 N} \times(N, N)$

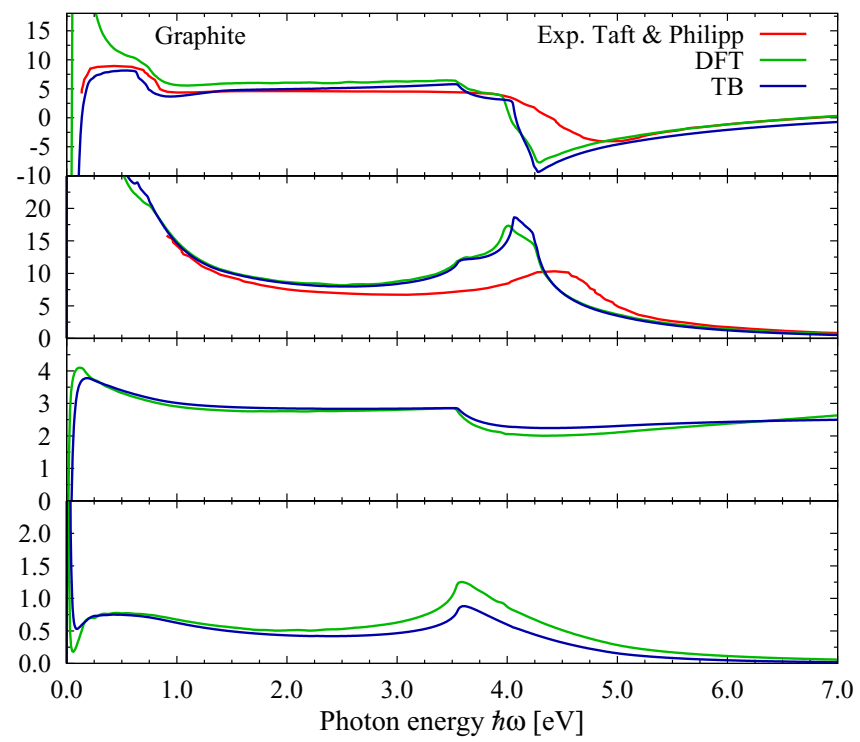

FIG. 2. Comparison between the permittivity of bulk hBN (left) and graphite (right) calculated using DFT and TB with a broadening of $\Gamma=5 \mathrm{meV}$. The two top panels show the real and imaginary parts of $\varepsilon_{\|}$and the bottom two panels show the real and imaginary parts of $\varepsilon_{\perp}$. The real parts of $\varepsilon_{\|}$and $\varepsilon_{\perp}$ have been shifted by 1.70 and 1.15 for bulk hBN and by 1.1 and 1.62 for graphite, to account for $\sigma$ electrons. For graphite we show also experimental results from Taft and Philipp [15] for the parallel component. 
for which $\frac{S}{2 N}$ is an integer. Here, $(N, N)$ should be understood as the finite building block consisting of $N$ sheets of $\mathrm{Gr}$ and $N$ sheets of hBN [cf. Fig. 1(b)] with the sheet permittivities given by the projected permittivities of the corresponding periodic structure $\{N, N\}$. We ignore the different environment of the sheets near the air boundaries. The choice of the topmost layer has a significant impact on the reflection [12], and therefore we average the reflection of the two possible configurations in all calculations. The TMM catches the field variation, since we, in effect, calculate the field strength in each sheet of the structure.

To calculate the conductivity due to the atoms belonging to the set $A=\left\{\alpha_{1}, \alpha_{2}, \ldots\right\}$ of atom indices $\alpha_{n}$, we need only change the MME in Eqs. (4) and (7). To this end, we define the projected $\mathrm{MME}$

$$
P_{m n}^{A}=\frac{1}{2} \sum_{\alpha \in A} \sum_{\beta}\left[c_{m, \alpha}^{*} c_{n, \beta}+c_{m, \alpha} c_{n, \beta}^{*}\right]\langle\alpha|\hat{p}| \beta\rangle,
$$

where, e.g., $c_{m, \alpha}$ refers to element $\alpha$ of eigenvector $m$ and $\langle\alpha|\hat{p}| \beta\rangle$ is the MME between the atomic $\pi$ wave functions $\alpha$ and $\beta$. If we sum over all such disjoint sets we get the MME for the entire structure $P_{m n}=\sum_{A} P_{m n}^{A}$. Now, we wish to define the projected conductivity $\sigma^{A}$ due to the atoms belonging to $A$ such that $\sigma=\sum_{A} \sigma^{A}$, that is, the projected conductivities should sum up to the total conductivity. Defining the squared projected MME as

$$
\begin{aligned}
M_{m n}^{A} & =\frac{1}{2} \sum_{B}\left(P_{m n}^{B *} P_{m n}^{A}+P_{m n}^{A *} P_{m n}^{B}\right) \\
& =\frac{1}{2} P_{m n}^{A} P_{m n}^{*}+\frac{1}{2} P_{m n}^{A *} P_{m n} \\
& =\operatorname{Re}\left(P_{m n}^{A} P_{m n}^{*}\right)
\end{aligned}
$$

and substituting $M_{m n}$ with $M_{m n}^{A}$ in Eq. (4), it is an easy task to show that $\sigma^{A}$ sum up to $\sigma$. To find the conductivity of a single sheet we identify the indices of the unit cell atoms belonging to this sheet, e.g., numbering the unit cell atoms from below, the bottom Gr sheet of Fig. 1(a) is described by the set $A_{1}=\{1,2\}$, the next $\mathrm{Gr}$ sheet by $A_{2}=\{3,4\}$, etc.

In Fig. 3, we show the projected sheet conductivity for a $\{7,7\}$ structure along with the conductivity of graphite and bulk hBN divided by 7 . We show only sheets $1-4$ (S1-S4) as, for symmetry reasons, S5 is identical to S3, etc. Sheets that are close to the boundary (S1) derive characteristics from the other material: The conductivity of hBN S1 has a peak at $4.1 \mathrm{eV}$ that coincides with the Gr sheets and also nonzero conductivity below the band gap that cannot be attributed to broadening, while Gr S1 has a peak at $5.7 \mathrm{eV}$ coinciding with the hBN sheets. We have checked that the projected conductivities sum up to the conductivity for the entire structure. Overall, close resemblance with the bulk conductivity is seen (also for the out-of-plane conductivity, which is not shown), suggesting that the response should be well described using bulk values. From the permittivities of the individual sheets it is a simple matter to calculate the reflection from the finite structure using the TMM and the Fresnel reflection coefficients between two anisotropic materials given by Eq. (A10) of Appendix.
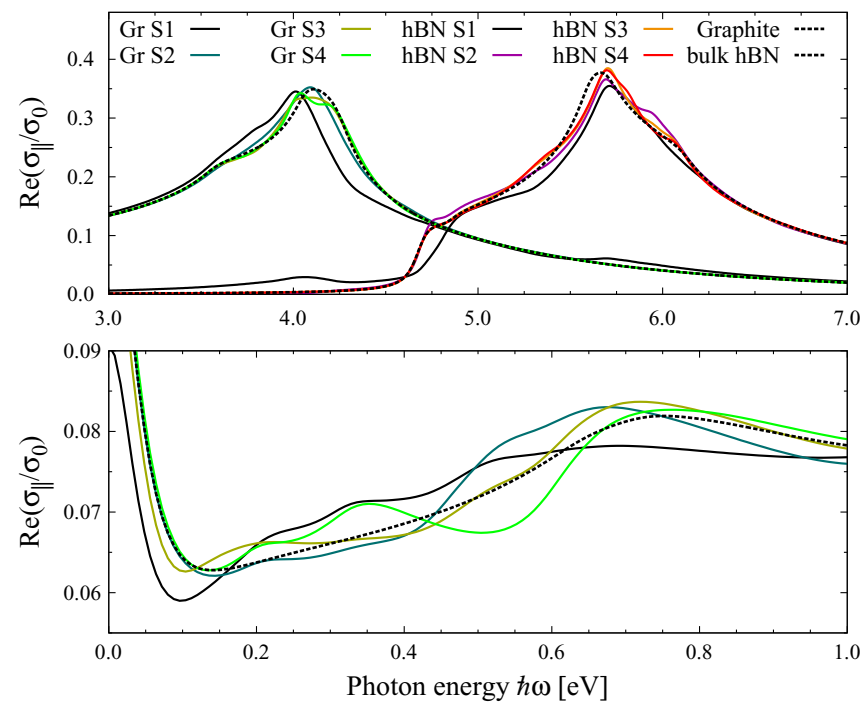

FIG. 3. Projected conductivity in the energy range 3-7 eV (top) and $0-1 \mathrm{eV}$ (bottom) of a $\{7,7\}$ structure along with the graphite/bulk $\mathrm{hBN}$ conductivity divided by 7 . The conductivity is given in units of the graphene minimum conductivity $\sigma_{0}=e^{2} / 4 \hbar$ [24].

\section{RESULTS AND DISCUSSION}

We have calculated $\varepsilon_{\|}$for $E_{F}=0.0 \mathrm{eV}$ for several periodic Gr-hBN heterostructures in order to investigate the convergence to EMT. We expect the TB calculations to converge to EMT as the number of sheets is increased, since deviations for thin layers are merely a measure of the importance of the Gr-hBN coupling $\left(\gamma_{1}^{\mathrm{CB}}\right)$ and of the difference between the individual constituents and their bulk counterparts (that is, graphite and bulk hBN), both of which vanish for increasing number of layers. Local field variations can be safely ignored in the parallel case as it has been argued above.

In Fig. 4, we show the results where the colored lines are full TB calculations using Eq. (4), the solid black curve is EMT based on permittivities of graphite and bulk hBN, and the dashed black curve is EMT based on graphene and monolayer hBN. Only for the $\{1,1\},\{3,3\}$, and $\{5,5\}$ cases we find significant discrepancies compared to EMT (bulk) while for $\{11,11\}$ the curves are difficult to distinguish on the chosen scale for both the real and imaginary part. For reference, we show in the bottom panel the absolute value of the deviation from EMT in percent for the imaginary part of $\varepsilon_{\|}$calculated as $\left|\operatorname{Im}\left(\varepsilon_{\|}^{\mathrm{TB}}-\varepsilon_{\|}^{\mathrm{EMT}}\right)\right| / \operatorname{Im}\left(\varepsilon_{\|}^{\mathrm{EMT}}\right)$. For the low-energy spectrum the convergence is slower than it may be expected. It is usually stated that EMT works well when the wavelength is much larger than the period of the structure [12], and this is true when applying EMT to calculations in which the phase of the incoming light is important. However, Fig. 4 represents a study of convergence in electronic structure for which the phase of the incoming light plays no role.

One may intuitively expect that EMT based instead on monolayer permittivities is in good agreement with the $\{1,1\}$ structure, but the dashed black curve of Fig. 4 shows that this is not the case. Monolayer graphene or $\mathrm{hBN}$ in a periodic structure behaves rather differently than its isolated counterparts, as one also finds if the projected conductivity 


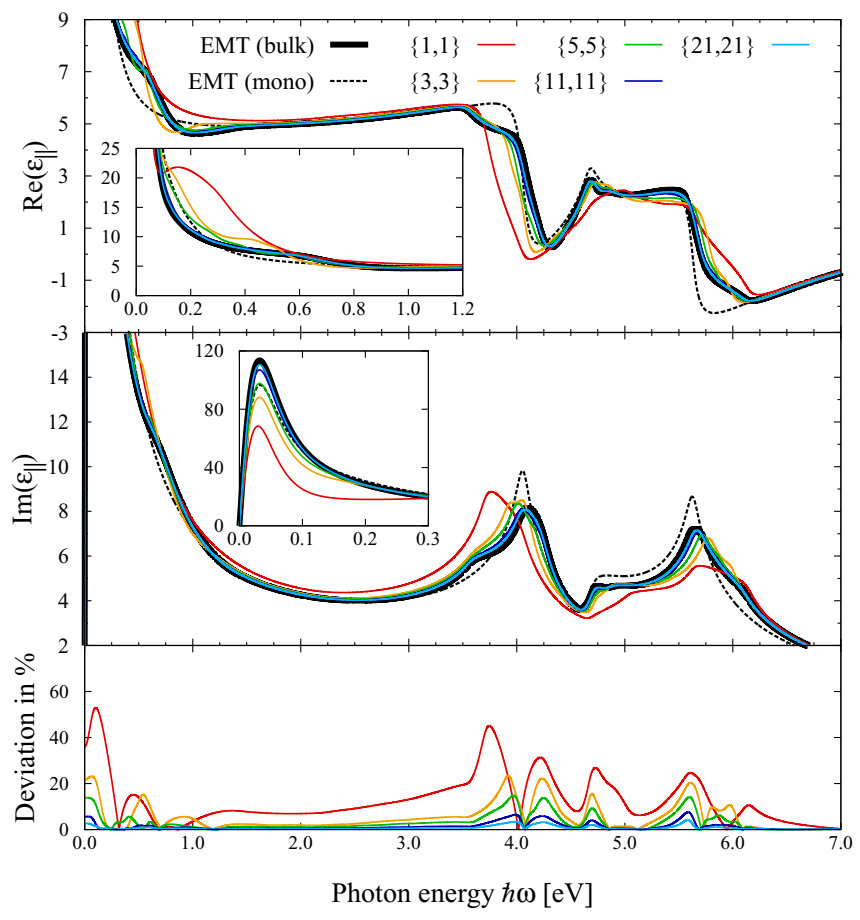

FIG. 4. Real (top) and imaginary (middle) part of the parallel permittivity $\varepsilon_{\|}$versus bulk and monolayer based EMT for $E_{F}=$ $0.0 \mathrm{eV}$. The insets show a zoom of the low-energy region. The bottom panel shows the absolute value of the deviation from EMT for the imaginary part in percent

of the $\mathrm{Gr}$ or $\mathrm{hBN}$ sheet of a $\{1,1\}$ structure is calculated (not shown). In the left panel of Fig. 5, we plot the equivalent of Fig. 4 for $E_{F}=0.5 \mathrm{eV}$. From this plot it is seen that the convergence to EMT (bulk) is slower than for $E_{F}=0.0 \mathrm{eV}$ in the same energy range and that, in particular for the $\{1,1\}$,
$\{3,3\}$, and $\{5,5\}$ structures, EMT predicts the incorrect sign of $\operatorname{Re}\left(\varepsilon_{\|}\right)$in some energy ranges.

One of the major deficiencies of EMT is the failure to include the coupling between the layers, thus, we suggest a simple improvement to EMT, which significantly improves the slower convergence in doped structures for low energies, and we refer in the following to this as EMTi. Many applications of graphene-based devices rely on the response in the regime close to $2 E_{F}$, thus, good convergence in this regime is important. We consider the effective medium to consist of three materials: bulk Gr, bulk hBN, and an interface layer. The interface layer is described as a finite slab of graphene on $\mathrm{hBN}$, such that the thickness of the slab is $2 c_{0}$, and its permittivity is calculated using the in-plane hopping parameters of graphite and bulk hBN as well as the carbon-boron coupling $\gamma_{1}^{\mathrm{CB}}$. We then average these three materials according to

$$
\begin{aligned}
& \varepsilon_{\|}^{\text {EMTi }}=\rho_{\mathrm{m}} \varepsilon_{\mathrm{m}}+\rho_{\mathrm{d}} \varepsilon_{\mathrm{d}}+\left(1-\rho_{\mathrm{m}}-\rho_{\mathrm{d}}\right) \varepsilon_{\text {interface }}, \\
& \frac{1}{\varepsilon_{\perp}^{\text {EMTi }}}=\frac{\rho_{\mathrm{m}}}{\varepsilon_{\mathrm{m}}}+\frac{\rho_{\mathrm{d}}}{\varepsilon_{\mathrm{d}}}+\frac{1-\rho_{\mathrm{m}}-\rho_{\mathrm{d}}}{\varepsilon_{\text {interface }}},
\end{aligned}
$$

where $\rho_{\mathrm{m}}$ and $\rho_{\mathrm{d}}$ are the fractions of metal and dielectric, respectively, and $\varepsilon_{\text {interface }}$ is the permittivity of the graphene/hBN slab. For a $\{5,5\}$ structure, for example, we would have $\rho_{\mathrm{m}}=0.3$ and $\rho_{\mathrm{d}}=0.3$. In this way the effect of coupling between $\mathrm{Gr}$ and $\mathrm{hBN}$ is to some extent included in the effective medium.

In the right panel of Fig. 5 we show the results of using EMTi. From the bottom panels showing the deviations is it clear that EMTi offers an improvement compared to ordinary EMT. The huge improvement on the $\{1,1\}$ structure is somewhat surprising, and suggest that in this structure the effects of interlayer coupling between $\mathrm{Gr}$ and $\mathrm{hBN}$ are more important than the effects of periodicity. For energies above $3 E_{F}$ we see only minor improvement using EMTi.
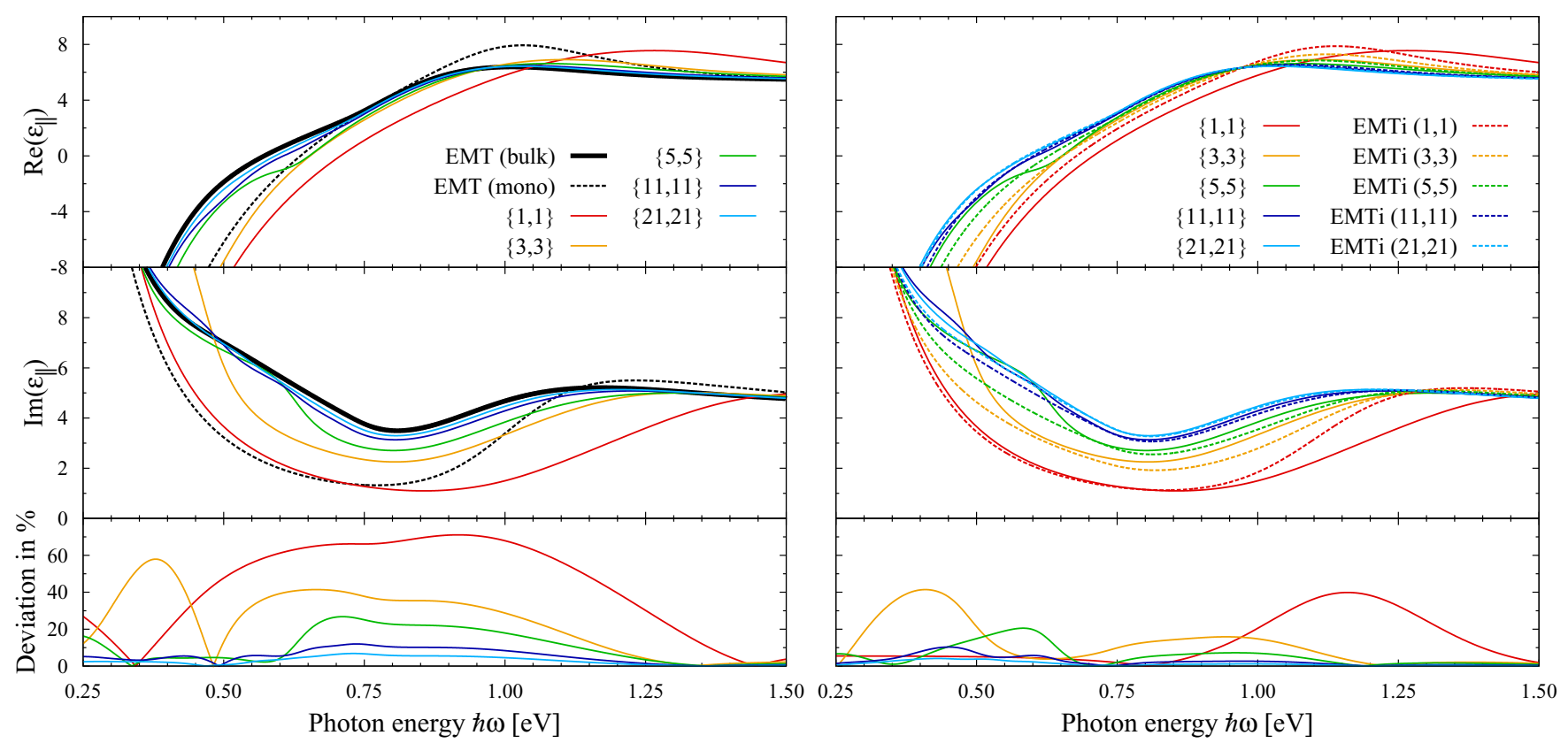

FIG. 5. Convergence to EMT (left) and EMTi (right) for $E_{F}=0.5 \mathrm{eV}$. The top panels show $\operatorname{Re}\left(\varepsilon_{\|}\right)$, the middle panels $\operatorname{Im}\left(\varepsilon_{\|}\right)$, and the bottom panels the absolute value of the deviation from EMT/EMTi for the imaginary part in percent. 

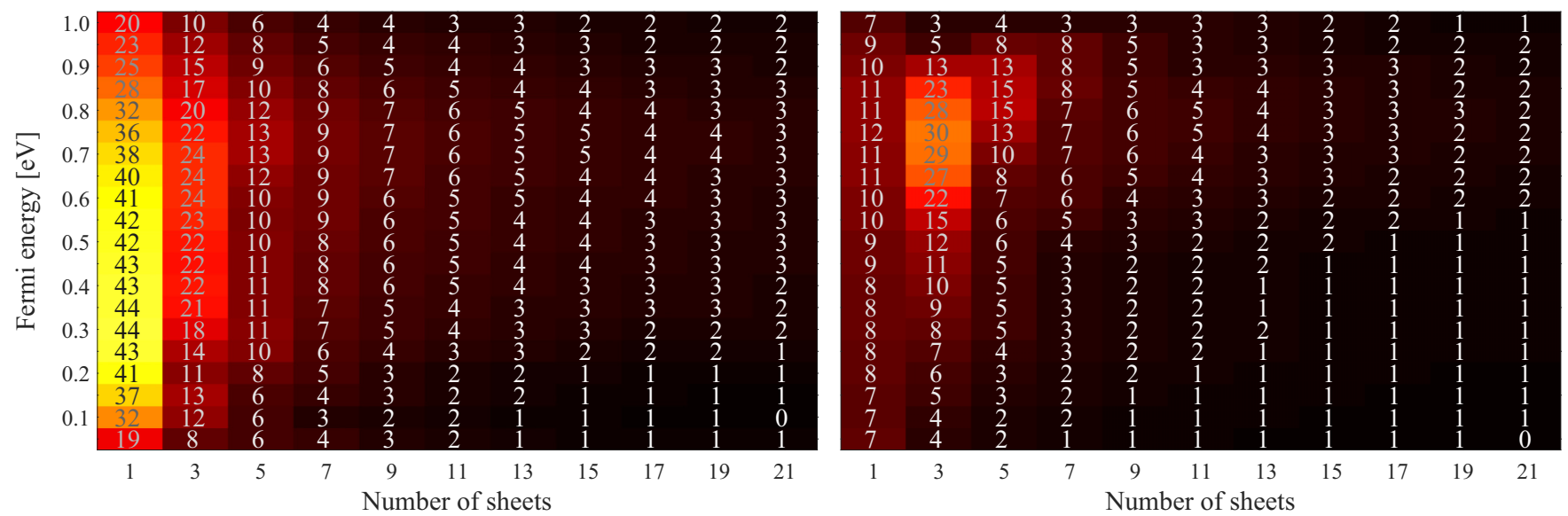

FIG. 6. Compilation of the averaged absolute value of the deviation of TB from EMT (left) and EMTi (right) in percent for a number of layers and Fermi levels. The average is taken over the energy interval $0 \mathrm{eV}$ to $3 E_{F}$.

To provide an overview of the error introduced using EMT, we show in the left and right panels of Fig. 6 a compilation of the averaged absolute value of the deviation for $\operatorname{Im}\left(\varepsilon_{\|}\right)$ in percent calculated using EMT and EMTi, respectively, for different structures and for a number of Fermi levels. The average is taken over the energy interval $0 \mathrm{eV}$ to $3 E_{F}$, since for higher energies the spectrum is only weakly dependent on $E_{F}$. We find that for all structures the error reaches a maximum at a certain value of $E_{F}$ both for EMT and for EMTi. This can be explained by comparing the band structure of the full structure with the band structures of the constituents on which EMT and EMTi are based. One finds then that for these particular Fermi levels, the averaging from 0 to $3 E_{F}$ includes predominantly transitions between states of the band structures that differ strongly. Large deviations from EMT are seen in the left panel, and these deviations exists over a broad range of $E_{F}$. Small structures in particular are consistently ill described in EMT. Great improvement is found by using EMTi, especially for the $\{1,1\}$ structure which is well described for all $E_{F}$, but also for larger structures. Generally, we find that although the deviation in some cases increases in EMTi, the large deviations are confined to a smaller interval of $E_{F}$.

For the perpendicular permittivity $\varepsilon_{\perp}$, we take into account the local field variation due to the stratified nature of the structure using the approach described in Sec. II. TMM does not allow us to directly calculate the permittivity, and so our figure of merit becomes the reflection. In Fig. 7, we show for $E_{F}=0.5 \mathrm{eV}$ the reflection $\left|r_{p}\right|^{2}$ of $p$-polarized light for two incident angles from the stacks $210 \times(1,1), 70 \times(3,3)$, $42 \times(5,5), 14 \times(15,15), 10 \times(21,21)$, and $6 \times(35,35)$ all of thickness $420 c_{0}$, and from the corresponding effective medium using EMT (black line). For $\theta_{\text {incident }}=0^{\circ}$ we probe only the parallel component of the permittivity tensor, thus, we expect rapid convergence to EMT for $\hbar \omega>3 E_{F}$ because we have seen that the permittivity converges rapidly in this range. For the stacks $210 \times(1,1), 70 \times(3,3)$, and $42 \times(5,5)$ the convergence is as expected in the shown energy range, while for $14 \times(15,15), 10 \times(21,21)$, and $6 \times(35,35)$ the convergence is only as expected in the low-energy regime while in the range 4-7 eV deviations from EMT that cannot be attributed to the EMT permittivity are seen. These deviations stem from interference effects due to multiple reflections at the interfaces between $\mathrm{Gr}$ and $\mathrm{hBN}$ layers that are not present in the EMT model, and these effects become increasingly important at smaller wavelengths (in the structure). To see this we plot also the reflection from a stack modeled as 12 layers of alternating graphite and bulk hBN each of thickness $35 c_{0}$, and we denote this stack as $6 \times(35 \mathrm{~B}, 35 \mathrm{~B})$ to emphasize that we use the bulk and not the sheet permittivities. The resemblance with the corresponding curve based on projected sheet permittivities confirms that the deviations from EMT are in fact caused by multiple reflections, and thereby that the permittivity has converged to the EMT value. From the insets it

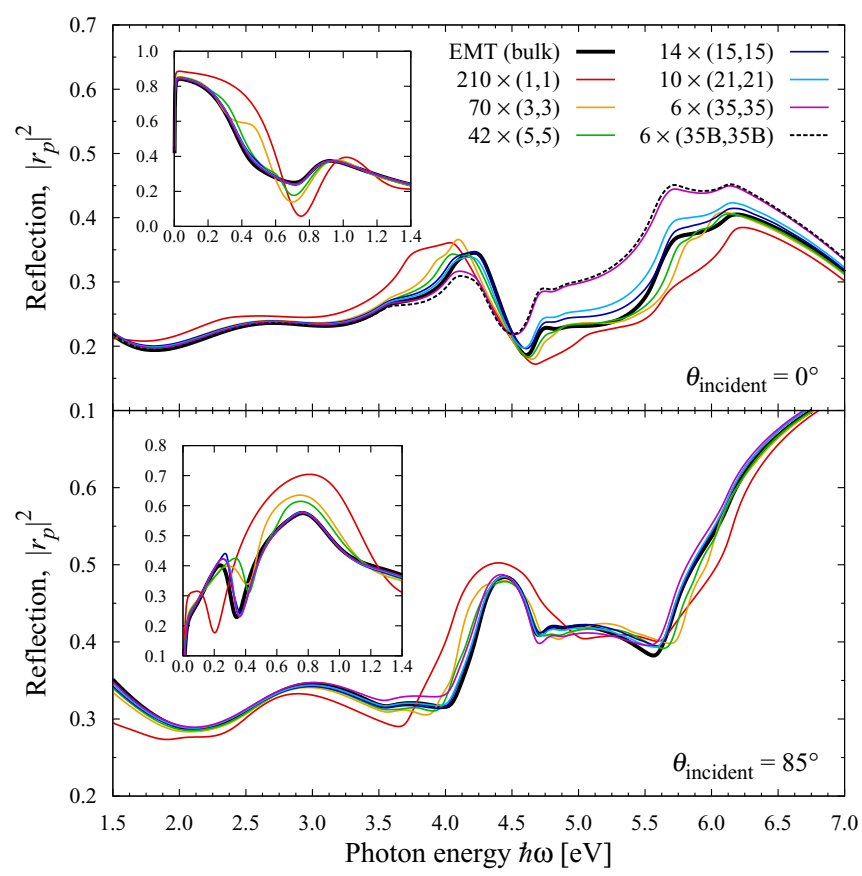

FIG. 7. Reflection of $p$-polarized light by the shown structures in an ambient of air at two angles of incidence and at $E_{F}=0.5 \mathrm{eV}$. The dashed black curve in the top panel is the reflection by a layered structure built using $\varepsilon$ of graphite and bulk hBN. The insets show the spectral range between $0 \mathrm{eV}$ and $1.4 \mathrm{eV}$. 


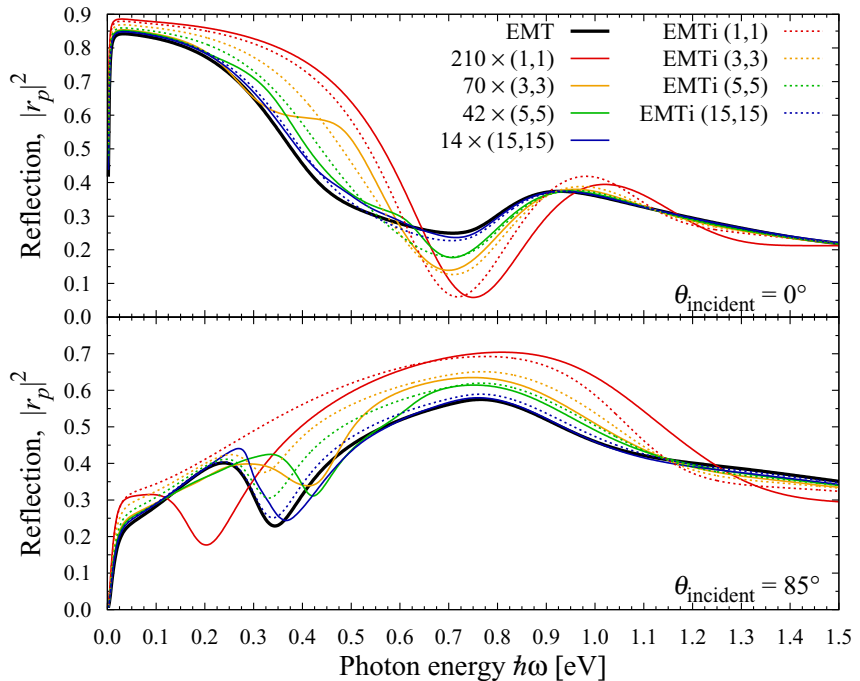

FIG. 8. Reflection of $p$-polarized light by the shown structures in an ambient of air at two angles of incidence and at $E_{F}=$ $0.5 \mathrm{eV}$, calculated using full TB permittivities and EMTi. For easy comparison, we show also the EMT result (solid black line).

is clear that the convergence is slow for $\hbar \omega<3 E_{F}$ as expected from the discussion of Fig. 5.

For $\theta_{\text {incident }}=85^{\circ}$ we probe primarily $\varepsilon_{\perp}$. At this angle of incidence we expect multiple reflections to give a much smaller contribution. In the bottom panel of Fig. 7, it can be seen that the reflection spectrum converges to the EMT spectrum calculated using Eq. (2) and that the convergence is as fast as for $\varepsilon_{\|}$. Small deviations are still seen in the range 4-7 eV due to multiple reflections, but they are much smaller than for $\theta_{\text {incident }}=0^{\circ}$. We show in Fig. 8 the reflection spectra calculated using the EMTi permittivities from Eq. (10) and compare with full TB-based spectra. It is clear that the convergence to EMTi is improved especially for normal incidence, where only $\varepsilon_{\|}$is probed, but also at $\theta_{\text {incident }}=85^{\circ}$ showing that also the perpendicular permittivity is described better in EMTi.

We have checked that our results remain valid also for asymmetric structures, but we remark that for structures with low absorption, that is, if the number of graphene sheets is low compared to the number of hBN sheets, multiple reflections become more important and may cause deviations from EMT if the number of layers is large. In particular, we have checked our results for a $15 \times(3,25)$ structure $(3 \mathrm{Gr}$ sheets per unit cell) and found that multiple reflections cause major deviations from EMT while for a $4 \times(3,25)$ good agreement with EMT is found.

Calculating projected permittivities and using the TMM to include the field variation is rather tedious. Therefore, one may be tempted to calculate simply the perpendicular response of the entire structure by using the MMEs Eq. (6) in Eqs. (4) and (7), thereby ignoring the field variation throughout the structure. We show in Fig. 9, that this method does not provide convergence to EMT and thus, that this simpler method of calculation is not feasible for the structures considered. The observed convergence is merely an expression for convergence in the electronic structure of the considered

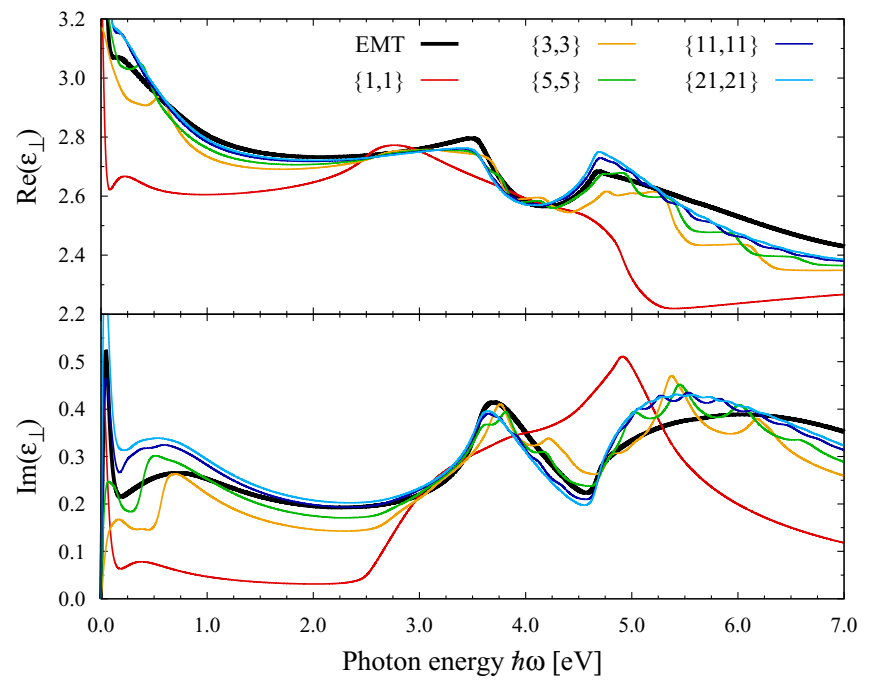

FIG. 9. Real (top) and imaginary (bottom) part of the perpendicular permittivity calculated using the MMEs Eq. (6) and thereby ignoring local field effects. This simpler method of calculation does not agree well with EMT, showing the importance of local field effects in the studied heterostructures.

structures, but this is generally not the same as convergence in optical response due to the importance of local field effects in heterostructures. We stress, however, that for asymmetric structures with $M \neq N$ ignoring the field variation is a reasonable approximation if one layer is much thicker than the other. We have run simulations for $\{3, M\}$ and $\{M, 3\}$ structures with $M=1,5,7,11,17,25$ (not shown) and found reasonable agreement with EMT for $M=11$ and above, although the convergence is slow.

Finally, we have calculated also the enhancement of spontaneous emission (the Purcell factor) of a dipole oriented parallel to and located a distance $h=20 \mathrm{~nm}$ above the surface of a $\mathrm{Gr} / \mathrm{hBN}$ stack using the expression in Ref. [28]. Both propagating and evanescent modes of $s$ and $p$ polarization contribute to the enhanced emission, although the huge Purcell factors that have been reported for HMMs $[28,29]$ are due to the coupling of evanescent modes of large parallel wavevector component $k_{x}$ with the HMM. When entering the structure the perpendicular wave-vector component will be $k_{z}^{s}=\sqrt{\varepsilon_{\|} k_{0}^{2}-k_{x}^{2}}$ and $k_{z}^{p}=\sqrt{\varepsilon_{\|} k_{0}^{2}-\left(\varepsilon_{\|} / \varepsilon_{\perp}\right) k_{x}^{2}}$ where $k_{0}=$ $\omega / c$ is the vacuum wave number, for $s$ - and $p$-polarized modes, respectively. If $k_{x}$ is large, both $k_{z}^{s}$ and $k_{z}^{p}$ can have large (positive) imaginary parts, and this is the case also in the hyperbolic regime where $\operatorname{Re}\left(\varepsilon_{\|}\right) \operatorname{Re}\left(\varepsilon_{\perp}\right)<0$ because of losses. The large imaginary part causes these modes to decay rapidly within the structure and therefore to see only the topmost sheets of the structure. Thus, even though the wavelength of the incoming light may be sufficiently large that EMT should hold, the rapid decay of the modes may cause EMT to break down far sooner than anticipated. We illustrate this in Fig. 10, where we plot the reflection coefficient of $p$-polarized evanescent modes of energy $\hbar \omega=0.30 \mathrm{eV}$ incident on the stacks $42 \times(5,5), 14 \times(15,15)$, and $6 \times(35,35)$ and on the corresponding effective medium, all with $E_{F}=0.5 \mathrm{eV}$. At this Fermi level these structures all exhibit hyperbolic behavior in 


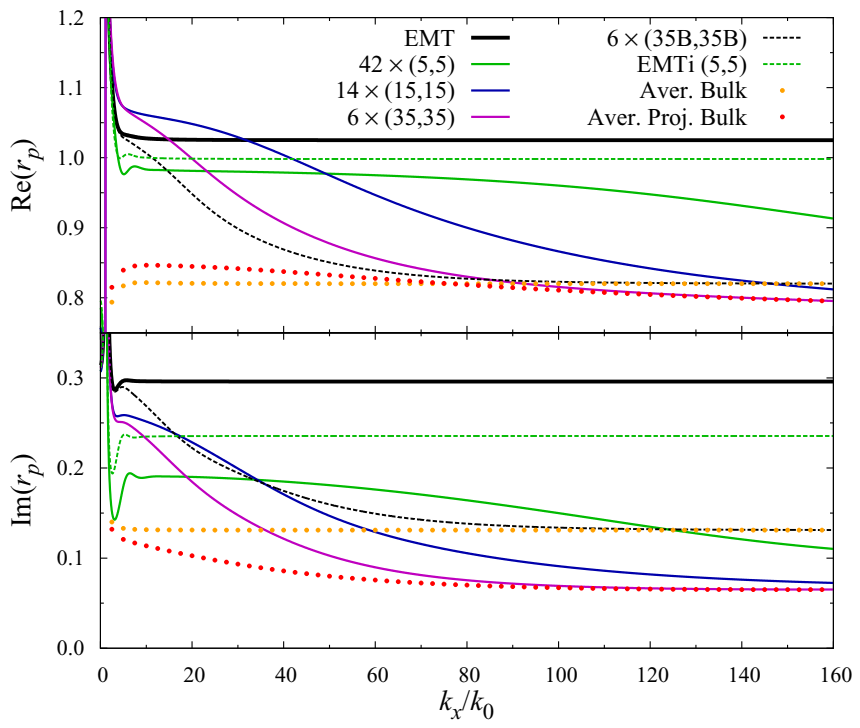

FIG. 10. Real and imaginary part of the reflection coefficient for $k_{x} / k_{0}$ up to 160 . The energy is $\hbar \omega=0.3 \mathrm{eV}$ and the Fermi level is $E_{F}=0.5 \mathrm{eV}$. The red dots represent the average of the reflection coefficient from a stack of graphite and bulk hBN both of thickness $420 c_{0}$. The same for the orange dots, but using the projected permittivities from a $\{35,35\}$ structure for the 17 topmost layers and the graphite/bulk $\mathrm{hBN}$ value for remaining layers.

the energy range $50 \mathrm{meV}$ to $0.6 \mathrm{eV}$. The pole near $k_{x}=k_{0}$ corresponds to a surface plasmon excitation typical for a $p$-polarized wave incident on a metal/dielectric interface. We remark that reflection coefficients greater than unity for modes of $k_{x}>k_{0}$ impose no violation of energy conservation due to their nonpropagating character in the medium of incidence.

For $k_{x} \approx 5 k_{0}$ EMT breaks down even for the very thin but still electronically converged $\{5,5\}$ based structures. That the explanation for this shall not be found in the multiple reflection scheme used to describe the discrepancies of Fig. 7, is seen from the dashed black curve, which represents the reflection from a stack based on layered graphite/bulk hBN just as the black dashed curve of Fig. 7. To confirm the hypothesis that only the topmost sheets contribute to the reflection for large $k_{x}$ we plot the averaged reflection coefficient from graphite and bulk hBN of thickness $420 c_{0}$ (orange dots). The agreement for large $k_{x}$ with the reflection from the $6 \times(35 \mathrm{~B}, 35 \mathrm{~B})$ stack, shows that indeed only the topmost sheets contribute to the reflection, since the orange dots represents the reflection from a nonlayered bulk structure. They both, however, suffer from similar deficiencies as EMT with incorrect limiting behavior. We calculate instead the averaged reflection coefficient from graphite and bulk hBN, but for the 17 topmost of the 420 sheets we use sheet $1-17(\mathrm{Gr})$ or sheet $36-52(\mathrm{hBN})$ of a $\{35,35\}$ calculation (red dotted curve). Doing this, we find exactly the correct limiting behavior, again confirming the hypothesis that only the topmost sheets contribute, but, more importantly, demonstrating the crucial role of effects near the interface in obtaining the correct limiting behavior. We have tested that these results are also valid for other energies within and outside the hyperbolic regime. These findings are well in line with other studies showing the importance of even single

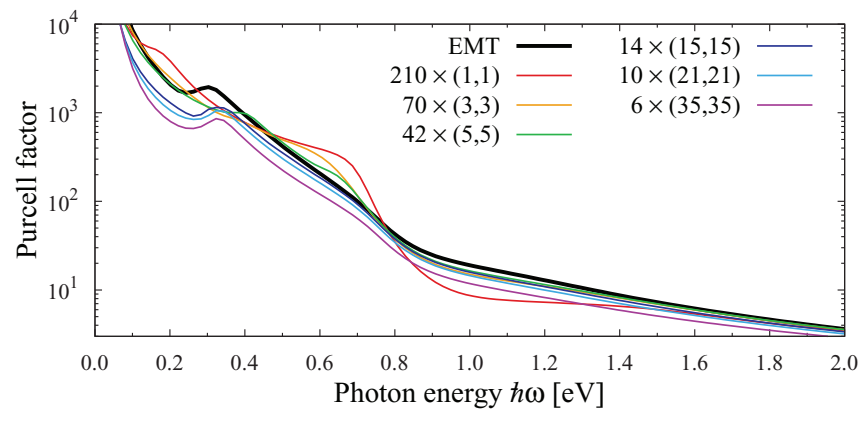

FIG. 11. Purcell factor for a dipole located $20 \mathrm{~nm}$ from and oriented parallel to the surface of the HMM. The Fermi level is $E_{F}=0.5 \mathrm{eV}$. For the large electronically converged structures shown, EMT overestimates the Purcell factor by up to a factor of 4 .

layers $[12,13]$ on transmission and reflection, but our results show also that the often ignored differences in electronic structure arising at an interface may in fact be very important.

For completeness we have included EMTi for the $42 \times$ $(5,5)$ structure. Improvement over EMT is certainly seen, but we remark that for thick layers EMTi suffers from the same deficiencies as EMT exactly because only the topmost layers, and not the layer interfaces, contribute.

The Purcell factor depends on the reflection coefficient of the structure at large $k_{x}$ and we therefore expect deviations from EMT for all structures. In Fig. 11, we present a calculation of the Purcell factor for a dipole-oriented parallel to and located $20 \mathrm{~nm}$ from the surface, showing as expected an increase within the hyperbolic region [28,29]. The increase in the Purcell factor depends critically on the damping and on the temperature and due to the relatively high broadening and temperature used in our calculations ( $50 \mathrm{meV}$ and $25 \mathrm{meV}$ ) we do not see enhancements as large as predicted by others [29]. For structures $210 \times(1,1), 70 \times(3,3)$ and $42 \times(5,5)$ the deviations from EMT are due to nonconverged permittivities, while the deviations observed for the converged structures are due to EMT not predicting correctly the reflection coefficient for high $k_{x}$ modes. The important conclusion to be made from our calculations, is the fact that EMT is a rather poor approximation for all structures in the energy range shown, overestimating the Purcell factor by up to a factor of 4 even for the converged structures.

\section{CONCLUSION}

We have investigated the validity of EMT in the extreme limit of metamaterials constructed from alternating layers of single atomic sheets of graphite and $\mathrm{hBN}$ and layers of up to 35 consecutive coupled sheets, and found that already for five sheets both $\varepsilon_{\|}$and $\varepsilon_{\perp}$ has converged. For structures in which the Gr part is doped, the convergence is slower. We offer a simple improvement to EMT, which greatly improves the convergence in the spectral range $0 \mathrm{eV}$ to $3 E_{F}$. For relatively thick layers of $\approx 10 \mathrm{~nm}$ (30 sheets) and more, multiple reflections at layer interfaces become important and causes EMT to break down for certain energies. In calculating the reflection of evanescent modes of large $k_{x}$, EMT always fails because only the topmost sheets of the structure contribute and this makes 
calculations very sensitive to the electronic details of these sheets. This causes EMT to overestimate the Purcell factor for the converged structures. In fact, we have shown that ignoring the atomistic effects caused by the boundaries of a structure can lead to quite different results. Our findings are important for the numerous works already using EMT and for future works in which EMT is used as a simple tool for providing a quick first estimate of material properties.

\section{ACKNOWLEDGMENTS}

The authors gratefully acknowledge the financial support from the Center for Nanostructured Graphene (Grant No. DNRF103) financed by the Danish National Research Foundation and from the QUSCOPE project financed by the Villum Foundation.

\section{APPENDIX: REFLECTION COEFFICIENTS}

In the following we outline the procedure used to determine the Fresnel reflection coefficients between two anisotropic materials characterized by the permittivity tensors

$$
\boldsymbol{\varepsilon}_{1}=\left(\begin{array}{ccc}
\varepsilon_{\|, 1} & 0 & 0 \\
0 & \varepsilon_{\|, 1} & 0 \\
0 & 0 & \varepsilon_{\perp, 1}
\end{array}\right), \quad \boldsymbol{\varepsilon}_{2}=\left(\begin{array}{ccc}
\varepsilon_{\|, 2} & 0 & 0 \\
0 & \varepsilon_{\|, 2} & 0 \\
0 & 0 & \varepsilon_{\perp, 2}
\end{array}\right) \text {. }
$$

The starting point is the wave equation in anisotropic media

$$
\nabla^{2} \mathcal{E}+k_{0}^{2} \varepsilon \cdot \mathcal{E}-\nabla(\nabla \cdot \mathcal{E})=0
$$

where $\boldsymbol{\varepsilon}$ is of the form Eq. (A1), $\mathcal{E}$ is the electric field and $k_{0}=\omega / c$. Assuming plane-wave solutions of the form $\mathcal{E}(\boldsymbol{r})=$ $\mathcal{E}_{0} e^{i \boldsymbol{k} \cdot \boldsymbol{r}}$ the wave equation takes the form

$$
-k^{2} \mathcal{E}_{0}+k_{0}^{2} \boldsymbol{\varepsilon} \cdot \mathcal{E}_{0}+\boldsymbol{k}\left(\boldsymbol{k} \cdot \mathcal{E}_{0}\right)=0 .
$$

For $s$ polarization the reflection coefficients are the same as in the isotropic case with permittivity $\varepsilon_{\|}$. For $p$ polarization the procedure is (i) determine the field directions in the anisotropic materials; (ii) express the incident, reflected and transmitted fields; and (iii) use the electromagnetic boundary conditions to determine the reflection/transmission coefficients. For $p$ polarization we use a result from Refs. [30,31] giving the non-normalized direction vector of the electric field inside an anisotropic material characterized by $\varepsilon_{\|}$and $\varepsilon_{\perp}$ as

$$
\boldsymbol{q}_{ \pm}=\frac{n^{p}}{k_{0}}\left[\frac{k_{x}}{\varepsilon_{\perp}} \hat{z} \mp \frac{k_{z}^{p}}{\varepsilon_{\|}} \hat{\boldsymbol{x}}\right],
$$

where $n^{p}$ is the effective index of refraction defined by

$$
n^{p}=\frac{\left|\boldsymbol{k}_{ \pm}^{p}\right|}{k_{0}}=\sqrt{\varepsilon_{\|}+\left(1-\frac{\varepsilon_{\|}}{\varepsilon_{\perp}}\right) \frac{k_{x}^{2}}{k_{0}^{2}}},
$$

with $\boldsymbol{k}_{ \pm}^{p}=k_{x} \hat{\boldsymbol{x}} \pm k_{z}^{p} \hat{z}$ and $k_{z}^{p}=\sqrt{k_{0}^{2} \varepsilon_{\|}-\left(\varepsilon_{\|} / \varepsilon_{\perp}\right) k_{x}^{2}}$. We notice that the effective refractive index reduces to the in-plane refractive index $\sqrt{\varepsilon_{\|}}$in the isotropic case and when $k_{x}=0$, corresponding to $s$-polarized light.

We can now formulate expressions for the incident, reflected, and transmitted fields in the two anisotropic materials. We assume light to be incident from material 1 and transmitted into material 2 , thus

$$
\begin{array}{rlrl}
\mathcal{E}_{i} & =\mathcal{E}_{0} \boldsymbol{q}_{-, 1} e^{i \boldsymbol{k}_{-, 1}^{p} \cdot \boldsymbol{r}}, & \mathcal{B}_{i} & =\frac{\mathcal{E}_{0}}{c k_{0}} n_{1}^{p} \hat{\boldsymbol{y}} e^{i \boldsymbol{k}_{-, 1}^{p} \cdot \boldsymbol{r}}, \\
\mathcal{E}_{r}=r \mathcal{E}_{0} \boldsymbol{q}_{+, 1} e^{i \boldsymbol{k}_{+, 1}^{p} \cdot \boldsymbol{r}}, & \mathcal{B}_{r}=r \frac{\mathcal{E}_{0}}{c k_{0}} n_{1}^{p} \hat{\boldsymbol{y}} e^{i \boldsymbol{k}_{+, 1}^{p} \cdot \boldsymbol{r}}, \\
\mathcal{E}_{t}=t \mathcal{E}_{0} \boldsymbol{q}_{-, 2} e^{i \boldsymbol{k}_{-, 2}^{p} \cdot \boldsymbol{r}}, & \mathcal{B}_{t}=t \frac{\mathcal{E}_{0}}{c k_{0}} n_{2}^{p} \hat{\boldsymbol{y}} e^{i \boldsymbol{k}_{-, 2}^{p} \cdot \boldsymbol{r}} .
\end{array}
$$

From the electromagnetic boundary conditions $\left(\mathcal{E}_{\|, 1}=\right.$ $\mathcal{E}_{\|, 2}$ and $\mathcal{B}_{\|, 1}=\mathcal{B}_{\|, 1}$ at the interface $z=0$ ) we obtain the Fresnel reflection and transmission coefficients between two anisotropic materials as

$$
\begin{gathered}
r_{p}=\frac{\varepsilon_{\|, 2} k_{z, 1}^{p}-\varepsilon_{\|, 1} k_{z, 2}^{p}}{\varepsilon_{\|, 2} k_{z, 1}^{p}+\varepsilon_{\|, 1} k_{z, 2}^{p}}, \\
t_{p}=\frac{2 \varepsilon_{\|, 2} k_{z, 1}^{p} n_{1}^{p} / n_{2}^{p}}{\varepsilon_{\|, 2} k_{z, 1}^{p}+\varepsilon_{\|, 1} k_{z, 2}^{p}} .
\end{gathered}
$$

The expression for $t_{p}$ reduces to Eq. (17) in Ref. [30] in the special isotropic case $\varepsilon_{\|, 1}=\varepsilon_{\perp, 1}=1$.
[1] Z. Liu, H. Lee, Y. Xiong, C. Sun, and X. Zhang, Far-field optical hyperlens magnifying sub-diffraction-limited objects, Science 315, 1686 (2007).

[2] M. Noginov, H. Li, Y. A. Barnakov, D. Dryden, G. Nataraj, G. Zhu, C. Bonner, M. Mayy, Z. Jacob, and E. Narimanov, Controlling spontaneous emission with metamaterials, Opt. Lett. 35, 1863 (2010).

[3] P. Shekhar, J. Atkinson, and Z. Jacob, Hyperbolic metamaterials: fundamentals and applications, Nano Converg. 1, 1 (2014).

[4] A. Poddubny, I. Iorsh, P. Belov, and Y. Kivshar, Hyperbolic metamaterials, Nat. Photon. 7, 948 (2013).

[5] K. Sreekanth, T. Biaglow, and G. Strangi, Directional spontaneous emission enhancement in hyperbolic metamaterials, J. Appl. Phys. 114, 134306 (2013).
[6] M. A. Othman, C. Guclu, and F. Capolino, Graphene-based tunable hyperbolic metamaterials and enhanced near-field absorption, Opt. Express 21, 7614 (2013).

[7] A. Andryieuski, A. V. Lavrinenko, and D. N. Chigrin, Graphene hyperlens for terahertz radiation, Phys. Rev. B 86, 121108 (2012).

[8] K. Sreekanth, A. De Luca, and G. Strangi, Negative refraction in graphene-based hyperbolic metamaterials, Appl. Phys. Lett. 103, 023107 (2013).

[9] L. Zhang, Z. Zhang, C. Kang, B. Cheng, L. Chen, X. Yang, J. Wang, W. Li, and B. Wang, Tunable bulk polaritons of graphenebased hyperbolic metamaterials, Opt. Express 22, 14022 (2014).

[10] R. Ning, S. Liu, H. Zhang, B. Bian, and X. Kong, A wide-angle broadband absorber in graphene-based hyperbolic metamaterials, Eur. Phys. J. Appl. Phys. 68, 20401 (2014). 
[11] T. Tumkur, Y. Barnakov, S. Kee, M. Noginov, and V. Liberman, Permittivity evaluation of multilayered hyperbolic metamaterials: Ellipsometry vs. reflectometry, J. Appl. Phys. 117, 103104 (2015).

[12] O. Kidwai, S. V. Zhukovsky, and J. Sipe, Effective-medium approach to planar multilayer hyperbolic metamaterials: Strengths and limitations, Phys. Rev. A 85, 053842 (2012).

[13] H. H. Sheinfux, I. Kaminer, Y. Plotnik, G. Bartal, and M. Segev, Subwavelength Multilayer Dielectrics: Ultrasensitive Transmission and Breakdown of Effective-Medium Theory, Phys. Rev. Lett. 113, 243901 (2014).

[14] J. Sławińska, I. Zasada, and Z. Klusek, Energy gap tuning in graphene on hexagonal boron nitride bilayer system, Phys. Rev. B 81, 155433 (2010).

[15] E. Taft and H. Philipp, Optical properties of graphite, Phys. Rev. 138, A197 (1965).

[16] A. Grüneis, C. Attaccalite, L. Wirtz, H. Shiozawa, R. Saito, T. Pichler, and A. Rubio, Tight-binding description of the quasiparticle dispersion of graphite and few-layer graphene, Phys. Rev. B 78, 205425 (2008).

[17] J. J. Mortensen, L. B. Hansen, and K. W. Jacobsen, Real-space grid implementation of the projector augmented wave method, Phys. Rev. B 71, 035109 (2005).

[18] W. Kohn and L. J. Sham, Self-consistent equations including exchange and correlation effects, Phys. Rev. 140, A1133 (1965).

[19] J. Yan, J. J. Mortensen, K. W. Jacobsen, and K. S. Thygesen, Linear density response function in the projector augmented wave method: Applications to solids, surfaces, and interfaces, Phys. Rev. B 83, 245122 (2011).

[20] A. MacDonald, S. Vosko, and P. Coleridge, Extensions of the tetrahedron method for evaluating spectral properties of solids, J. Phys. C: Solid State 12, 2991 (1979).
[21] W. Paszkowicz, J. Pelka, M. Knapp, T. Szyszko, and S. Podsiadlo, Lattice parameters and anisotropic thermal expansion of hexagonal boron nitride in the 10-297.5 k temperature range, Appl. Phys. A 75, 431 (2002).

[22] G. Constantinescu, A. Kuc, and T. Heine, Stacking in Bulk and Bilayer Hexagonal Boron Nitride, Phys. Rev. Lett. 111, 036104 (2013).

[23] B. Sachs, T. Wehling, M. Katsnelson, and A. Lichtenstein, Adhesion and electronic structure of graphene on hexagonal boron nitride substrates, Phys. Rev. B 84, 195414 (2011).

[24] T. G. Pedersen, C. Flindt, J. Pedersen, A.-P. Jauho, N. A. Mortensen, and K. Pedersen, Optical properties of graphene antidot lattices, Phys. Rev. B 77, 245431 (2008).

[25] T. G. Pedersen, Analytic calculation of the optical properties of graphite, Phys. Rev. B 67, 113106 (2003).

[26] L. G. Johnson and G. Dresselhaus, Optical properies of graphite, Phys. Rev. B 7, 2275 (1973).

[27] G. E. Jellison Jr, J. D. Hunn, and H. N. Lee, Measurement of optical functions of highly oriented pyrolytic graphite in the visible, Phys. Rev. B 76, 085125 (2007).

[28] A. M. DaSilva, Y.-C. Chang, T. Norris, and A. H. MacDonald, Enhancement of photonic density of states in finite graphene multilayers, Phys. Rev. B 88, 195411 (2013).

[29] I. V. Iorsh, I. S. Mukhin, I. V. Shadrivov, P. A. Belov, and Y. S. Kivshar, Hyperbolic metamaterials based on multilayer graphene structures, Phys. Rev. B 87, 075416 (2013).

[30] R. J. Gehr, G. L. Fischer, R. W. Boyd, and J. Sipe, Nonlinear optical response of layered composite materials, Phys. Rev. A 53, 2792 (1996).

[31] J. J. Saarinen and J. Sipe, A green function approach to surface optics in anisotropic media, J. Mod. Opt. 55, 13 (2008). 